\title{
Einschätzungen zum elektronischen Rezept - eine Querschnittstudie unter Apothekern in Deutschland
}

\section{Assessing Electronic Prescription: A Cross-sectional Study of Pharmacists in Germany}

\section{(웅 $\odot$}

\author{
Autoren \\ Christoph Strumann(D), Birger Möller, Jost Steinhäuser
}

Institute

Institute of Family Medicine, Universitätsklinikum SchleswigHolstein - Campus Lübeck, Lübeck, Deutschland

\section{Schlüsselwörter}

Mixed-Methods, e-Health, e-Rezept, Telemedizin, Digitalisierung, Versorgungsforschung

\section{Keywords}

mixed methods, e-health, e-prescription, telemedicine, digitization, health care research

online publiziert $\quad 23.06 .2021$

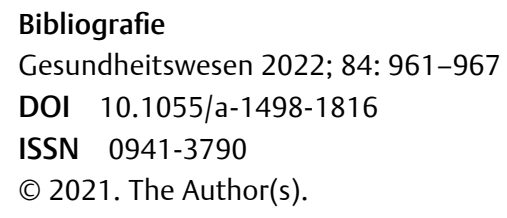
Creative Commons Attribution-NonDerivative-NonCommercial-License, permitting copying and reproduction so long as the original work is given appropriate credit. Contents may not be used for commercial purposes, or adapted, remixed, transformed or built upon. (https://creativecommons. org/licenses/by-nc-nd/4.0/)

Georg Thieme Verlag KG, Rüdigerstraße 14,

70469 Stuttgart, Germany

\section{Korrespondenzadresse}

Christoph Strumann

Institute of Family Medicine

Universitätsklinikum Schleswig-Holstein - Campus Lübeck

Ratzeburger Allee 160

23538 Lübeck

Deutschland

c.strumann@uni-luebeck.de

$\circledast$ Z Zusätzliches Material finden Sie unter https://doi. org/10.1055/a-1498-1816

\section{ZUSAMMENFASSUNG}

Hintergrund Mit dem Modellprojekt GERDA („Geschützter eRezept-Dienst der Apotheken“) wurde von der Landesapotheker- kammer (LAK) und dem Landesapothekerverband (LAV) in Baden-Württemberg die Möglichkeit geschaffen, Rezepte im Rahmen von Online-Sprechstunden über das von der Kassenärztlichen Vereinigung Baden-Württemberg betriebene telemedizinische Portal „docdirekt“, zu verordnen. Vor diesem Hintergrund war das Ziel der Studie, Barrieren und fördernde Faktoren aufseiten der Apotheken für die Beteiligung an einer Versorgung, die eine elektronische Verordnung von Rezepten beinhaltet, zu explorieren und zu priorisieren. Auf Basis dieser Determinanten wurden Empfehlungen zur Optimierung einer erfolgreichen Implementierung ähnlicher Versorgungsangebote abgeleitet.

Methode Es wurde ein Mixed-Methods-Design gewählt, um die Determinanten zu explorieren und zu priorisieren. Im ersten Schritt wurden Determinanten für die Beteiligung an einem System, welches die elektronische Verordnung von Rezepten ermöglicht mithilfe von Einzelinterviews mit an docdirekt beteiligten Teleärzten, niedergelassenen Hausärzten und Apothekern exploriert. Anschließend wurden diese Determinanten in einem zweiten Schritt durch eine quantitative Befragung von Apothekern priorisiert.

Ergebnisse Von den 523 an der Umfrage teilgenommenen Apothekern war mehr als die Hälfte bereit sich an einem ERezept-System zu beteiligen, 8,5\% schließen eine zukünftige Beteiligung aus. Insgesamt konnten 18 Determinanten für die Beteiligung an einem System, welches die elektronische Verordnung von Rezepten ermöglicht, exploriert werden. Der Schutz der freien Apothekenwahl wurde als wichtigster Aspekt für eine Beteiligung identifiziert. Gefolgt von der Möglichkeit einer Korrekturfunktion bei E-Rezepten (um z. B. Retaxierungen oder Medikationsfehlern zu vermeiden), der Integration des E-Rezepts in die bestehende Apotheken-EDV sowie dem gesetzlichen Ausschluss von direkten Verträgen mit Versandapotheken. Zeitersparnisse und eine ggf. höhere Vergütung wurden dagegen als weniger relevant bewertet.

Schlussfolgerung Insgesamt gab über die Hälfte der befragten Apotheken an, sich an einem E-Rezept-System beteiligen zu wollen. Für die ab Januar 2022 geplante flächendeckende Einführung von E-Rezepte kann der Einbezug der identifizierten fördernden Faktoren vonseiten der Politik, Softwareentwickler und Verbände eine wichtige Rolle bei der Implementierung spielen. 


\section{ABSTRACT}

Background The GERDA (“Protected e-prescription service for pharmacies") project of the Chamber of Pharmacists (LAK) and Pharmacists Association (LAV) of the federal state of BadenWuerttemberg provided the opportunity to prescribe medications within the telemedical portal "docdirekt" that is operated by the Association of Statutory Health Insurance Physicians of Baden-Wuerttemberg. Against this background, the aim of the study was to explore and prioritize barriers and enablers among pharmacists to participate in a medical supply system that includes electronic prescriptions (e-prescriptions). Based on these determinants, recommendations for optimizing the successful implementation of similar care offers were derived.

Method A mixed methods design was chosen to explore and prioritize the determinants. In the first step, determinants for participation in an electronic prescribing system were explored by means of individual interviews of docdirekt tele-physicians, primary care physicians and pharmacists. In a second step, these determinants were prioritized through a quantitative survey of pharmacists.
Results Out of the 523 pharmacists that answered the questionnaire, more than half were willing to participate in an eprescription system, while $8.5 \%$ excluded future participation. A total of 18 determinants for the e-prescription system participation could be explored. The protection of the free pharmacy choice for the patients was identified as the most important determinant, followed by the option of a correction function for e-prescriptions (e. g. to avoid retaxing or medication errors), the integration of the e-prescription into the existing pharmacy IT system and the statutory exclusion of direct contracts with online pharmacies. Time savings and possibly higher remunerations were rated as less relevant.

Conclusion More than half of the pharmacies surveyed stated that they wanted to participate in an e-prescription system. Widespread introduction of e-prescriptions in planned for January 2022. Successful implementation of this move will be facilitated if the identified determinants are taken into consideration by politics, software developers and associations.

\section{Einleitung}

Mit der Einführung des elektronischen Rezepts (E-Rezept) ab Januar 2022 soll eine papierlose Verschreibung von Arznei- oder Hilfsmitteln in Deutschland ermöglicht werden. Das E-Rezept soll die Lücke in der telemedizinischen Betreuung von Patienten schließen, wenn diese im Rahmen einer Videosprechstunde das Rezept in elektronischer Form erhalten können [1]. Darüber hinaus soll das E-Rezept eine Schlüsselrolle dabei spielen, die unterschiedlichen Akteure im Gesundheitswesen (besonders Ärzte und Apotheker) im Hinblick auf die gemeinsame Versorgung des Patienten digital zu vernetzen und den Weg für weitere E-Health-Funktionen zu ebnen [2].

Elektronische Verordnungssysteme haben sich in vielen Ländern bereits vollständig etabliert [3] und es konnten neben Zeit- und Kostenersparnissen aufseiten der Ärzte, Apotheker und Patienten [4] auch klinische Auswirkungen wie z. B. genauere Dosierungen sowie eine Reduzierung von Medikationsfehlern und unerwünschter Wechsel- oder Nebenwirkungen demonstriert werden [5]. In Deutschland sollte das E-Rezept ursprünglich im Jahr 2004 flächendeckend eingeführt werden. Neben technischen Problemen beim Aufbau der benötigten Infrastruktur durch die Telematik, führten Widerstände aufseiten aller Beteiligten zu Verzögerungen [6].

Für den Erfolg einer E-Health-Anwendung wie ein E-Rezept ist die Akzeptanz der Beteiligten (Ärzte, Apotheker und Patienten) entscheidend [7, 8]. Eine Akzeptanz erfolgt bei Innovationen zumeist nicht spontan, sondern in einem mehrstufigen Prozess, der sich über einen bestimmten Zeitraum erstreckt und eine Reihe von Handlungen beinhaltet [9]. In allen Stufen des Prozesses wird mittels Informationsbeschaffung oder dem Testen der Innovation versucht, bestehende Unsicherheiten bzgl. des neuen Angebots zu reduzieren.

Mittlerweile werden in über fünf Modellprojekten in Deutschland Erfahrungen zum E-Rezept gesammelt, die Erkenntnisse zu möglichen Barrieren und fördernden Faktoren bei der Akzeptanz aufzeigen sollen. Mit dem Modellprojekt GERDA („Geschützter eRezept-Dienst der Apotheken“) wurde von der Landesapothekerkammer (LAK) und dem Landesapothekerverband (LAV) in BadenWürttemberg die Möglichkeit geschaffen, Rezepte im Rahmen einer Videosprechstunde auszustellen, ohne dabei die freie Entscheidung des Patienten einzuschränken, in welcher der (beteiligten) Apotheken das Rezept eingelöst wird. Die Integration von GERDA erfolgte dabei in das Angebot der ausschließlichen Fernbehandlung „docdirekt“ der Kassenärztlichen Vereinigung BadenWürttemberg (KVBW), in welchem das E-Rezept für gesetzlich Krankenversicherte erprobt werden sollte [10].

Vor diesem Hintergrund war das Ziel der Studie, Barrieren und fördernde Faktoren aufseiten der Apotheken für die Beteiligung an einer Versorgung, die eine elektronische Verordnung von Rezepten beinhaltet, zu explorieren und zu priorisieren. Auf Basis dieser Determinanten können Empfehlungen zur Optimierung einer erfolgreichen Implementierung ähnlicher Versorgungsangebote abgeleitet werden.

\section{Methoden}

Es wurde ein Mixed-Methods-Design gewählt, um die Determinanten zu explorieren und zu priorisieren. Im ersten Schritt wurden Determinanten für die Beteiligung an einem System, welches die elektronische Verordnung von Rezepten ermöglicht mithilfe von Einzelinterviews mit an docdirekt beteiligten Teleärzten, niedergelassenen Hausärzten und Apothekern exploriert. Anschließend wurden diese in einem zweiten Schritt durch eine quantitative Befragung von Apothekern priorisiert.

\section{Einzelinterviews}

Die Rekrutierung der Teilnehmenden für die qualitative Erhebung fand durch die KVBW (an docdirekt beteiligte Teleärzte), der LAK 
(Apotheker) sowie dem Institut für Allgemeinmedizin in Lübeck (Hausärzte) statt. Die Befragung der Probanden zu fördernden Faktoren und Barrieren bei der Beteiligung an einem System zur elektronischen Verschreibung von Rezepten wurde mithilfe eines zuvor entwickelten, teilstandardisierten Interviewleitfadens durchgeführt.

\section{Fragebogen}

Für die quantitative Befragung wurde auf Grundlage der Einzelinterviews ein Fragebogen erstellt. Dieser beinhaltete 18 aus den vorangegangenen Interviews ausgewählte explorierte Determinanten. Die Befragten konnten die Determinanten hinsichtlich des Einflusses auf ihre Bereitschaft, sich an einer Versorgung zu beteiligen, die ein E-Rezept mit beinhaltet, jeweils mittels der Ausprägungen „positiv“, „, /- Null“, und „negativ“ bewerten. Zusätzlich wurden neben den soziodemographischen Daten der Probanden auch allgemeine Fragen zur Einschätzung rund um die Entwicklung des E-Rezepts sowie deren generelle Bereitschaft zur Beteiligung an einem E-Rezept-System erhoben (Fragebogen siehe Online-Supplement). Der Fragebogen wurde nicht formal validiert, aber im Rahmen einer Pilotierung auf Klarheit und Verständlichkeit geprüft.

Der Link zur Onlineumfrage wurde an 2 verschiedenen Gruppen von Apotheken verschickt. Als erste Gruppe wurden alle Apotheken aus Baden-Württemberg eingeladen. Die zweite Gruppe bestand aus Apotheken aus ganz Deutschland, die sich im (öffentlich zugänglichen) Versandhandelsregister des Bundesinstituts für Arzneimittel und Medizinprodukte (BfArM) für den Internethandel registriert haben [11]. In Baden-Württemberg wurde die Umfrage von der LAK an 2.429 öffentliche Apotheken am 25. September 2020 und als Reminder am 2. und 14. Oktober 2020 per E-Mail verschickt. An die 1.200 Versandhandel-Apotheken wurde der Link am 20. und als Reminder am 27. November 2020 und 4. Dezember 2020 verschickt.

\section{Statistische Analyse}

Zu den einzelnen Determinanten wurden die jeweiligen Anteile derjenigen berechnet, die diese mit „positiv“ bewerteten. Die Reihenfolge in der Priorisierung wurde anschließend auf Basis der Anteile bestimmt. Zur Beschreibung der Zusammenhänge zwischen verschiedenen Eigenschaften der Befragten und der Bereitschaft sich an einer Versorgung zu beteiligen, die ein E-Rezept mit beinhaltet, wurde eine multivariate Regressionsanalyse (Ordinale Logistische Regression) durchgeführt. Die Beteiligungsbereitschaft wurde mit der Frage operationalisiert, ob sich die Apotheker bereits an einem E-Rezept-System beteiligt haben. Die Antwortmöglichkeiten („Ja.“, „Nein. Aber sobald es möglich ist, werde ich mich beteiligen.“, „Nein. Aber sobald es möglich ist, würde ich mich vielleicht beteiligen." oder „Nein. Ich will mich auch in Zukunft nicht daran beteiligen.") wurden als ordinal skalierte Beteiligungsbereitschaft herangezogen. Die berücksichtigten Eigenschaften waren Variablen zur Soziodemografie (Geschlecht, Alter, Stadt), Apotheke (Anzahl an Mitarbeitenden, Entfernung zur nächsten Apotheke, DSL-Anschluss, Registrierung als Versandhandel-Apotheke) und medizinischen Versorgung (Einschätzung der medizinischen Versorgung, einer festen Stammapotheke für die Patienten und die Zusammenarbeit mit Ärzten vor Ort). Die statistischen Analysen wurden mit STATA 15 (StataCorp LLC, College Station, TX, USA) durchgeführt.

\section{Ergebnisse}

\section{Exploration der Determinanten}

Es wurden insgesamt 26 Interviews durchgeführt. Darunter waren 10 Teleärzte, die an docdirekt beteiligt waren (7 Allgemeinmediziner und 3 Pädiater), 9 Apotheker und 7 niedergelassene Hausärzte (6 Allgemeinmediziner und ein Internist). Das durchschnittliche Alter der Teilnehmenden betrug 54,3 Jahre (SD = 8,9; min: 30 J., max: 71 J.), der Anteil der weiblichen Teilnehmenden betrug $33 \%$.

Insgesamt konnten 18 Determinanten für die Beteiligung an einem System, welches die elektronische Verordnung von Rezepten ermöglicht, exploriert werden.

\section{Quantitative Ergebnisse}

Die an insgesamt 3.629 Emailadressen verschickten Einladungen führten zu 523 ausgefüllten Fragebögen (Rücklaufquote: 14\%). Alle Rückläufer waren gültig und konnten in die Analyse eingeschlossen werden. - Tab. 1 zeigt u. a. die soziodemographischen Merkmale der Studienteilnehmenden, Einschätzung zur Entwicklung und Möglichkeiten rund um das E-Rezept, sowie Beurteilung der medizinischen Versorgung.

Das durchschnittliche Alter der befragten Apotheker betrug 51,4 Jahre (SD = 10,4; min: 19 J., max: 71 J.). Die Hälfte von ihnen waren weiblich (50,7\%), hatten ihre Apotheke in einer subjektiv als ländlich bewerteten Region (56,0\%) und im Mittel wurden 9,6 Mitarbeitende beschäftigt. 75 Apotheken hatten sich bereits an einem E-Rezept-System beteiligt und 199 würden es sofort tun, falls dies möglich wäre. Somit gaben insgesamt über die Hälfte der Befragten an, dass sie sich an einem E-Rezept-System beteiligt haben oder dieses würden. Mehr als ein Drittel (38,6\%) war noch unentschlossen und 8,5\% der Apotheker schlossen eine zukünftige Beteiligung aus. Während einige Teilnehmende Chancen im E-Rezept sahen die Attraktivität des Services oder den Wert der eigenen Apotheke zu erhöhen, wurden die Angebote der Online-Versandapotheken im Hinblick auf das eigene Geschäftsmodell überwiegend als sehr bedrohlich eingestuft. Eine feste Stammapotheke für Patienten sowie der Austausch mit Ärzten vor Ort wurde als sehr wichtig bewertet, während die allgemeine medizinische Versorgung vor Ort im Mittel als gut empfunden wurde.

\section{Bewertung der Determinanten}

Für jede der 18 Determinanten wurde der Anteil derjenigen berechnet, die diese als einen positiven Einfluss bewerteten. Auf dieser Basis wurde für alle Teilnehmenden eine Rangordnung der Determinanten erstellt ( $\triangleright$ Tab. 2).

Den höchsten Anteil erzielte mit 95\% der Schutz der freien Apothekenwahl. Gefolgt von der Möglichkeit einer Korrekturfunktion bei E-Rezepten (um z. B. Retaxierungen oder Medikationsfehler zu vermeiden), der Integration des E-Rezepts in die bestehende Apotheken-EDV sowie dem gesetzlichen Ausschluss von direkten Verträgen mit Versandapotheken. Auch die Existenz einer analogen Rückfallebene, falls es Schwierigkeiten mit der Software geben sollte, sowie die störungsfreie Funktion der nötigen Software für das E-Rezept wurden als hoch priorisiert. Einen mittleren Rang belegten Aspekte der Investitionen, der Bedienbarkeit der nötigen Software, die Möglichkeit eines Zugriffs auf den Medikationsplan, Mitarbeiterschulungen und des Datenschutzes. Die Möglichkeit, dass 
Tab. 1 Deskriptive Statistiken.

\begin{tabular}{|c|c|}
\hline Variable & Mittelwert / n (Standardabweichung) \\
\hline \multicolumn{2}{|l|}{ Soziodemografie } \\
\hline Alter & $51,4 / 515(10,4)$ \\
\hline Weiblich & $261 / 515(50,7)^{*}$ \\
\hline Männlich & $250 / 515(48,5)^{*}$ \\
\hline Divers & $4 / 515(0,8)^{*}$ \\
\hline Ländlich & $291 / 520(56,0)^{*}$ \\
\hline Städtisch & $229 / 520(44,0)^{*}$ \\
\hline \multicolumn{2}{|l|}{ Apotheke } \\
\hline DSL-Anschluss & $363 / 521(69,7)^{*}$ \\
\hline Registrierte Internet-Apotheke & $71 / 523(13,6)^{*}$ \\
\hline Anzahl Mitarbeiter & $9,6 / 513(5,2)$ \\
\hline Autominuten zur nächsten & $4,5 / 498(4,1)$ \\
\hline Hausarztpraxis & $2,3 / 425(2,1)$ \\
\hline \multicolumn{2}{|l|}{ Haben Sie sich bereits an einem E-Rezept-System beteiligt? } \\
\hline Ja. & $75 / 518(14,5)^{*}$ \\
\hline Nein. Aber sobald es möglich ist, werde ich mich beteiligen. & $199 / 518(38,4)^{*}$ \\
\hline Nein. Aber sobald es möglich ist, würde ich mich vielleicht beteiligen. & $200 / 518(38,6)^{*}$ \\
\hline Nein. Ich will mich auch in Zukunft nicht daran beteiligen. & $44 / 518(8,5)^{*}$ \\
\hline \multicolumn{2}{|l|}{ Einschätzung zur Entwicklung und Möglichkeiten durch das E-Rezept } \\
\hline Wie finden Sie die Möglichkeiten eines E-Rezepts? (5: sehr gut, ... , 1 sehr schlecht) & $3,2 / 493(1,2)$ \\
\hline $\begin{array}{l}\text { Ich sehe in E-Rezepten eine Chance, die Attraktivität der Services meiner Apotheke für meine Patienten zu } \\
\text { erhöhen. (5: sehr hoch, ..., } 1 \text { sehr gering) }\end{array}$ & $3,5 / 516(1,2)$ \\
\hline Mit der Bestandsanzeige meiner Ware könnte ich neue Patienten werben. (5: sehr hoch, ..., 1 sehr gering) & $3,1 / 502(1,3)$ \\
\hline $\begin{array}{l}\text { Die Teilnahme am E-Rezept würde eine Wertsteigerung meiner Apotheke bedeuten. (5: sehr hoch, ... , } 1 \\
\text { sehr gering) }\end{array}$ & $3,2 / 495(1,3)$ \\
\hline $\begin{array}{l}\text { Wie gut fühlen Sie sich von den Organen der Selbstverwaltung (Landesapothekerkammer, usw.) über das } \\
\text { E-Rezept informiert? (5: sehr gut, ..., } 1 \text { sehr schlecht) }\end{array}$ & $2,7 / 479(1,1)$ \\
\hline $\begin{array}{l}\text { Wie beurteilen Sie die Angebote der Online-Versandapotheken im Hinblick auf Ihr eigenes Geschäftsmo- } \\
\text { dell? (5: sehr bedrohlich, ..., } 1 \text { überhaupt nicht bedrohlich) }\end{array}$ & $4,5 / 520(0,7)$ \\
\hline \multicolumn{2}{|l|}{ Beurteilung der Versorgung } \\
\hline Stammapotheke für Patienten (5: sehr wichtig, ..., 1 sehr unwichtig) & $4,5 / 520(0,6)$ \\
\hline Austausch mit Ärzten vor Ort (5: sehr wichtig, ..., 1 sehr unwichtig) & $4,7 / 516(0,6)$ \\
\hline Medizinische Versorgung vor Ort (5: sehr gut, ..., 1 sehr schlecht) & $3,9 / 522(0,9)$ \\
\hline
\end{tabular}

Patienten das E-Rezept an Dritte (z. B. Angehörige) übermitteln können, wurde von knapp der Hälfte der Apotheken als relevant für ihre Beteiligungsbereitschaft angesehen und belegte damit einen eher unteren Rang (15 von 18). Zeitersparnisse und eine höhere Vergütung wurden ebenfalls geringer bewertet, sowie die Möglichkeit eine Onlineberatung für Patienten anbieten zu können.

\section{Regressionsmodell zur Beteiligungsbereitschaft}

Zur Bewertung der Sensitivität der Ergebnisse der Ordinalen Logistischen Regression gegenüber den Modellspezifikationen, wurden 4 Modelle mit ansteigender Komplexität geschätzt. Im Modell mit der höchsten Komplexität wurde ein Interaktionseffekt zwischen der Einschätzung der Medizinische Versorgung und der Wichtigkeit des Austausches mit Ärzten vor Ort spezifiziert (Modell (4)). Die Ergebnisse sind in $>$ Tab. 3 abgebildet.
Insgesamt waren die Schätzer der Regressionskoeffizienten stabil über die verschiedenen Modellspezifikationen. Nur der Einfluss der Stadt änderte sich und wurde insignifikant, nachdem Apothekeneigenschaften mitberücksichtigt werden. Größere Apotheken und für den Internethandel registrierte Apotheken waren eher bereit sich zu beteiligen sowie solche, die darin eine Chance sahen, die Attraktivität des Services für ihre Patienten zu erhöhen. Die Beteiligungsbereitschaft der Apotheker war umso größer, je wichtiger sie den Austausch mit den Ärzten vor Ort einstuften. Dieser Effekt war dabei umso stärker, je schlechter die allgemeine medizinische Versorgung vor Ort eingeschätzt wurde (Interaktionseffekt).

\section{Diskussion}

In der vorliegenden Analyse konnten Barrieren und fördernde Faktoren aufseiten der Apotheken für die Beteiligung an einer Versor- 
> Tab. 2 Bewertung der Determinanten.

\begin{tabular}{|l|l|l|}
\hline $\mathbf{R g}$ & Determinante & Anzahl / n (Anteil in \%) mit positivem Einfluss \\
\hline 1 & Freie Apothekenwahl bleibt geschützt & $494 / 520(95,0)$ \\
\hline 2 & Möglichkeit einer Korrekturfunktion & $490 / 521(94,0)$ \\
\hline 3 & Integration in die bestehende Apotheken-EDV & $476 / 519(91,7)$ \\
\hline 4 & Gesetzliches Verbot direkter Verträge mit Versandapotheken & $466 / 519(89,8)$ \\
\hline 5 & Analoge Rückfallebene & $465 / 520(89,4)$ \\
\hline 6 & Störungsfreie Software & $450 / 518(86,9)$ \\
\hline 7 & Übernahme der notwendigen Investitionen & $439 / 518(84,7)$ \\
\hline 8 & Transparenz über Kosten für notwendige Investitionen & $437 / 521(83,9)$ \\
\hline 9 & Möglichkeit des Zugriffs auf Medikationsplan & $431 / 521(82,7)$ \\
\hline 10 & Einfach Bedienbarkeit der Software & $423 / 519(81,5)$ \\
\hline 11 & Möglichkeit umfassender Mitarbeiterschulung & $411 / 520(79,0)$ \\
\hline 12 & Empfang des E-Rezepts auf möglichst vielen Endgeräten & $365 / 517(70,6)$ \\
\hline 13 & Datenschutzrechtlich sicheres System & $366 / 520(70,4)$ \\
\hline 14 & Patienten sind über die Nutzung von E-Rezepten informiert & $314 / 519(60,5)$ \\
\hline 15 & Möglichkeit, das E-Rezept an Dritte (z. B. Angehörige) zu übermitteln & $287 / 520(55,2)$ \\
\hline 16 & Zeitersparnis durch das E-Rezept & $272 / 520(52,3)$ \\
\hline 17 & Höhere Vergütung für ein E-Rezept als für ein „normales“ Rezept & $247 / 519(47,6)$ \\
\hline 18 & Möglichkeit der Onlineberatung & $233 / 519(44,9)$ \\
\hline
\end{tabular}

gung, die eine elektronische Verordnung von Rezepten beinhaltet, exploriert und priorisiert werden.

Die als am wichtigsten erachteten Aspekte für eine Beteiligung betrafen vor allem den Schutz des Geschäftsmodells der Apotheken, wie die Sicherung der freien Apothekenwahl und dem Makelverbot für elektronische Verordnungen. Die Gefahr, die von dem E-Rezept durch eine starke Zunahme des Versandhandels für das eigene Geschäftsmodell ausgeht, wurde von fast allen Apothekern als sehr hoch bewertet. Bisher müssen die Rezepte postalisch an die Versandhändler geschickt werden, bevor die Ware an den Patienten versendet werden kann. Das E-Rezept kann dagegen vom Patienten direkt elektronisch weitergeleitet werden. Laut der SEMPORA Apothekenmarktstudie 2020 würde für $35 \%$ der Patienten die Attraktivität für den Apothekenversandhandel durch das E-Rezept steigen und weniger als die Hälfte (44\%) würde das E-Rezept exklusiv in einer Apotheke vor Ort einlösen [12]. In den letzten 10 Jahren konnte ein Rückgang der Apotheken durch Filialschließungen von 11 \% beobachtet werden [13]. Das E-Rezept könnte diesen Trend weiter beschleunigen [14]. Inwiefern dies negative Auswirkungen auf die gesamte Versorgungslage haben kann, ist bisher noch nicht untersucht worden.

Die Apotheken, die sich für den Versandhandel registriert haben, haben generell eine höhere Bereitschaft sich an einem ERezept-System zu beteiligen. Das E-Rezept bietet aber auch für die nicht am Versandhandel beteiligten Apotheken Möglichkeiten ihre Wettbewerbsfähigkeit gegenüber dem Versandhandel zu stärken sowie auch Möglichkeiten für eine Verbesserung der allgemeinen Versorgungslage [15]. Die Apotheken könnten durch die für die Übermittlung des E-Rezepts vorgesehene Telematikinfrastruktur (TI) [16] eine direkte digitale Verbindung mit den Ärzten nutzen, um ihre Beratungskompetenz für den Patienten vor Ort auszubauen. Einige der befragten Apotheken scheinen in diesen Koopera- tionsmöglichkeiten eine Chance zu sehen; diejenigen, die den Austausch mit den Ärzten als wichtig einstuften, hatten auch eine höhere Beteiligungsbereitschaft. In Regionen, in denen die allgemeine medizinische Versorgung als schlechter eingeschätzt wurde, war dieser Effekt noch stärker. Eine Erklärung hierfür könnte sein, dass gerade in solchen Regionen die Beratungskompetenz der Apotheken eine größere Rolle für die allgemeine Versorgung der Patienten hat als in Regionen mit einer besseren medizinischen Versorgung. Das E-Rezept bietet hierbei eine neue Möglichkeit der Vernetzung und des Austausches mit den Ärzten vor Ort.

Fast 5,7\% aller stationärer Aufnahmen in Deutschland gehen auf unerwünschte Wirkungen von Medikamenten zurück, von denen über die Hälfe als vermeidbar eingestuft werden. Besonders betroffen sind hiervon Patienten, die über 65 Jahre alt sind [17]. Ein Drittel aller über 65-jährigen nimmt 5 oder mehr Medikamente ein, zwischen 75 und 85 Jahren sogar mehr als acht [18]. Durch die Polymedikation erhöht sich neben der Anzahl an möglichen Arzneimittelinteraktionen auch die Zeit, welche pro Patienten bei der ärztlichen Beratung aufgewendet werden muss. Fehlerhafte, unvollständige oder schlecht lesbare Verschreibungen werden durch die Eindeutigkeit des E-Rezepts reduziert. Eine mögliche Anknüpfung des E-Rezepts an einen elektronischen Medikationsplan [16] würde es Ärzten und Apothekern darüber hinaus ermöglichen die Medikation eines Patienten gemeinsam im Blick zu haben. Hiervon könnten besonders Patienten profitieren, die zusätzlich zu den ärztlich verschriebenen Medikamenten auch Arzneimittel im Rahmen der Selbstmedikation einnehmen [19]. Besonders im Hinblick auf eine zu erwartende Zunahme der Multimorbidität und daraus resultierender Polypharmazie wird dieser Aspekt in der Zukunft für die Patientensicherheit sicherlich noch an Bedeutung gewinnen [20]. 
> Tab. 3 Ordinales Logit-Modell zur Beteiligungsbereitschafta.

\begin{tabular}{|c|c|c|c|c|c|}
\hline \multicolumn{2}{|l|}{ Variable } & $(1)$ & $(2)$ & (3) & (4) \\
\hline \multicolumn{6}{|l|}{ Soziodemografie } \\
\hline \multicolumn{2}{|l|}{ Alter } & $-0,008$ & $-0,001$ & 0,005 & 0,007 \\
\hline \multicolumn{2}{|l|}{ Weiblich } & $-0,164$ & $-0,002$ & $-0,156$ & $-0,184$ \\
\hline \multicolumn{2}{|l|}{ Städtisch } & $0,369^{*}$ & 0,08 & 0,023 & 0,038 \\
\hline \multicolumn{6}{|l|}{ Apotheke } \\
\hline \multicolumn{2}{|l|}{ DSL-Anschluss } & & 0,174 & 0,191 & 0,182 \\
\hline \multicolumn{2}{|c|}{ Registrierte Internet-Apotheke } & & $0,827^{* *}$ & $0,740 * *$ & $0,713^{*}$ \\
\hline \multicolumn{2}{|l|}{ Anzahl Mitarbeiter } & & $0,092^{* *}$ & $0,081^{* *}$ & $0,081^{* *}$ \\
\hline \multicolumn{2}{|c|}{ Nächste Apotheke (min) } & & $-0,028$ & $-0,029$ & $-0,026$ \\
\hline \multicolumn{6}{|c|}{ Beurteilung der Versorgung } \\
\hline \multicolumn{2}{|c|}{ Stammapotheke für Patienten } & & & $-0,158$ & $-0,159$ \\
\hline \multicolumn{2}{|c|}{ Austausch mit Ärzten vor Ort } & & & $0,375^{*}$ & $0,363^{*}$ \\
\hline \multicolumn{2}{|c|}{ Medizinische Versorgung vor Ort } & & & $-0,015$ & $-0,005$ \\
\hline \multicolumn{6}{|c|}{$\begin{array}{l}\text { Einschätzung zu Möglichkeiten durch das } \\
\text { E-Rezept }\end{array}$} \\
\hline \multicolumn{2}{|c|}{ Chance die Attraktivität des Services zu erhöhen } & & & $0,424 * *$ & $0,428^{* *}$ \\
\hline \multicolumn{6}{|l|}{ Interaktionseffekt } \\
\hline \multicolumn{2}{|c|}{$\begin{array}{l}\text { medizinische Versorgung \& Austausch mit Ärzten } \\
\text { vor Ort }\end{array}$} & & & & $-0,359^{*}$ \\
\hline \multirow{3}{*}{ Schwellenwerte } & $\alpha_{1}$ & $-2,766^{* *}$ & $-1,616^{* *}$ & 0,86 & 0,941 \\
\hline & $\alpha_{2}$ & $-0,454$ & 0,804 & $3,426^{* *}$ & $3,530 * *$ \\
\hline & $\alpha_{3}$ & $1,455^{* *}$ & $2,878^{* *}$ & $5,573^{* *}$ & $5,685^{* *}$ \\
\hline \multicolumn{2}{|l|}{ LOGLIKE } & $-567,9$ & $-538,7$ & $-522,1$ & $-520,0$ \\
\hline \multicolumn{2}{|c|}{ Akaike Info Criterion (AIC) } & 1147,0 & 1097,4 & 1072,2 & 1070,0 \\
\hline \multicolumn{2}{|c|}{$\mathrm{R}^{2}$-MacFadden (in \%) } & 1,81 & 4,61 & 7,55 & 7,92 \\
\hline
\end{tabular}

Finanzielle Aspekte, mögliche Zeitersparnis durch das E-Rezept oder Bedenken wegen des Datenschutzes spielen dagegen eine geringere Rolle für die Akzeptanz der Apotheken. Dies lässt vermuten, dass viele Apotheker sich von finanziellen und datenschutzrechtlichen Risiken nicht abschrecken lassen ihre Wettbewerbsfähigkeit durch das E-Rezept zu stärken. Die relativ geringere Bewertung von potentiellen Zeitersparnissen kann darauf hindeuten, dass die Möglichkeiten durch das E-Rezept für einen verbesserten Service die erwarteten Effizienzsteigerungen im Betriebsablauf überwiegen. Laut einer Apokix-Umfrage des IFH Köln aus dem Jahre 2019 gaben $90 \%$ der Apotheken an, dass sie den Kunden auch digitale Dienstleistungen anbieten müssen, um erhebliche Wettbewerbsnachteile vermeiden zu können. Diesen Ausbau der Dienstleistungen in Eigenregie zu bewerkstelligen sahen jedoch $87 \%$ als Herausforderung an [21].

Zur Stärkung der Bereitschaft der Apotheken sich an einem ERezept-System zu beteiligen, sollte vor allem ein gesetzlicher Schutz der Apotheken vor Ort sowie vor sie umgehenden Verträgen (z. B. durch Rezeptmakler) forciert werden. Um die Nutzung der Möglichkeiten des E-Rezepts zur Stärkung der Wettbewerbsfähigkeit der vor Ort Apotheken hervorzuheben, sollten Verbände auf eine stärkere lokale Vernetzung zwischen den Leistungserbrin- gern (vor allem Ärzte und Apotheken) sowie den Ausbau der Beratungsfunktion der vor Ort Apotheken drängen. Darüber hinaus könnten klare Empfehlungen an die Mitglieder zum Anschluss an das E-Rezept-System sowie damit verbundene weitere digitale Dienstleistungen die Bereitschaft der vor Ort Apotheken erhöhen.

\section{Stärken und Schwächen}

Die Studie priorisiert Barrieren und fördernde Faktoren aufseiten der Apotheken für die Beteiligung an einer Versorgung, die eine elektronische Verordnung von Rezepten beinhaltet. Darüber hinaus konnten weitere Assoziationen mit der Beteiligungsbereitschaft exploriert werden. Auf Basis dieser Ergebnisse wurden Empfehlungen zur Optimierung einer erfolgreichen Implementierung des E-Rezepts abgeleitet.

Eine Stärke dieser Studie ist, dass die Ergebnisse sowohl auf Apotheken basieren, die sich für den Versandhandel registriert haben und solche, die sich nicht registriert haben. Zwischen beiden Gruppen konnten zwar Unterschiede in der allgemeinen Akzeptanz zum E-Rezept gefunden werden, jedoch gab es keine Unterschiede in der Priorisierung der explorierten Determinanten. Darüber hinaus wurden Apotheken befragt, die in ländlichen und städtischen Gebieten ansässig sind. Auch wenn die Einteilung in städtischen und 
ländlichen Regionen auf einer subjektiven Einschätzung der Apotheker/innen beruht, zeigt sie doch, dass die Ergebnisse der Studie auf einem breiten Spektrum der Apothekerschaft basieren. Eine Schwäche der Arbeit ist, dass wir nicht dafür kontrollieren konnten, welche Rolle oder Funktion die Teilnehmenden in ihrer Apotheke hatten. Da die große Mehrheit der Befragten ( 95\%) auch die strategischen Fragen zum Potential des E-Rezepts (z. B. Mit der Bestandsanzeige meiner Ware könnte ich neue Patienten werben.) beantworten konnten, gehen wir davon aus, dass die meisten befragten Apotheker/innen in unternehmerisch-strategischen Entscheidungen involviert sind und daher eine bedeutsame Rolle für die Implementierung des E-Rezepts in Deutschland spielen. Ein Selektionsbias der Studienprobanden kann aufgrund des eher geringen Rücklaufs (14\%) nicht ausgeschlossen werden, wodurch die Ergebnisse vorsichtig interpretiert werden müssen. Weitere Analysen zeigten jedoch, dass die Priorisierung robust für verschiedene Subgruppen (z. B. mit unterschiedlichen Beteiligungsbereitschaften, Stadt vs. Land, Geschlecht) ist.

\section{Schlussfolgerung}

Insgesamt gab über die Hälfte der befragten Apotheken an, sich an einem E-Rezept-System beteiligen zu wollen. Auch wenn ab Januar 2022 Ärzte verpflichtet werden E-Rezepte auszustellen und Apotheken verpflichtet sind Arzneimittel auf Grundlage von E-Rezepten abzugeben, kann der Implementierungsprozess optimiert werden, um die Potenziale für die Verbesserung der Versorgung vollständig auszuschöpfen. Hierbei kann die Adressierung der Determinanten der Apotheken vonseiten der Politik, Technik-/Sotfwareanbieter und Verbände eine wichtige Rolle spielen.

\section{Danksagung}

Die Autoren danken allen an der Erhebung Beteiligten für Ihre Unterstützung, insbesondere der Kassenärztlichen Vereinigung Baden-Württemberg, dem Landesapothekerverband Baden-Württemberg sowie der Landesapothekerkammer Baden-Württemberg.

\section{Interessenkonflikt}

Die Autoren geben keinen Interessenkonflikt an. Die Studie wurde finanziell durch die Landesapothekerkammer Baden-Württemberg und das Ministerium für Soziales und Integration Baden-Württemberg unterstützt. Die Studie erfolgte weisungsunabhängig.

\section{Literatur}

[1] Krüger-Brand HE. Elektronisches Rezept; Im zweiten Anlauf soll es klappen. Deutsches Ärzteblatt 2020; 117: 4

[2] Dietzel GT. Von eEurope 2002 zur elektronischen Gesundheitskarte Chancen für das Gesundheitswesen. Deutsches Arzteblatt 2002; 99: 1417-1419
[3] Samadbeik M, Ahmadi M, Sadoughi F, Garavand A. A Copmarative Review of Electronic Prescription Systems: Lessons Learned from Developed Countries. J Res Pharm Pract 2017; 6: 3-11

[4] Lanham AE, Cochran GL, Klepser DG. Electronic prescriptions: opportunities and challenges for the patient and pharmacist. Advanced Health Care Technologies 2016; 2: 12

[5] Bhavsar GP, Probst JC, Bennett K], Hardin JW, Qureshi Z. Communitylevel electronic prescribing and adverse drug event hospitalizations among older adults. Health Informatics Journal 2019; 25: 661-675

[6] Caumanns J. Zur Diskussion: Stand der Digitalisierung im deutschen Gesundheitswesen. Zeitschrift für Evidenz, Fortbildung und Qualität im Gesundheitswesen 2019; 143: 22-29

[7] Scott Kruse C, Karem P, Shifflett K, Vegi L, Ravi K, Brooks M. Evaluating barriers to adopting telemedicine worldwide: A systematic review. J Telemed Telecare 2016; 24: 4-12

[8] Gagnon M-P, Nsangou É-R, Payne-Gagnon J, Grenier S, Sicotte C. Barriers and facilitators to implementing electronic prescription: a systematic review of user groups' perceptions. J Am Med Inform Assoc 2013; 21: 535-541

[9] Rogers EM. Diffusion of Innovations, vol. Fourth EditionNew York: The Free Press; 2003

[10] von Solodkoff M, Strumann C, Steinhäuser J. Akzeptanz von Versorgungsangeboten zur ausschließlichen Fernbehandlung am Beispiel des telemedizinischen Modellprojekts „docdirekt“: ein Mixed-Methods Design. Gesundheitswesen 2021; 83: 186-194

[11] Übersicht der Internet-Apotheken https://versandhandel.dimdi.de/ pdfs/vhr-apo.pdf (abgerufen am 01.12.2020)

[12] Brodtkorb AT. SEMPORA Apothekenmarktstudie 2020. SEMPORA Consulting gmbh 2020

[13] Bundesvereinigung Deutscher Apothekerverbände (ABDA). Die Apotheke: Zahlen Daten Fakten 2020 https://www.abda.de/fileadmin/ user_upload/assets/Pressetermine/2020/TdA_2020/ABDA_ZDF_2020_ Brosch.pdf (abgerufen am 12.01.2021)

[14] Dr Kaske. Marketingberatung. E-Rezept-Studie 2019. https://drkaske. de/studien/e-rezept-studie-2019/ (abgerufen am 12.01.2021)

[15] Goundrey-Smith S. The Connected Community Pharmacy: Benefits for Healthcare and Implications for Health Policy. Front Pharmacol 2018; 9: 1352

[16] Systemspezifisches Konzept E-Rezept https://www.vesta-gematik.de/ standard/formhandler/324/gemSysL_eRp_V1_0_0_CC6.pdf (abgerufen am 14.01.2021)

[17] Köberle U, Stammschulte T, Gundert-Remy U, Pitzer M, Bräutigam K. Erfassung und Bewertung von Medikationsfehlern. Bundesgesundheitsblatt - Gesundheitsforschung - Gesundheitsschutz 2018; 61: 1066-1074

[18] Schwabe U, Ludwig W-D. Arzneiverordnungen 2019 im Überblick. In: Arzneiverordnungs-Report 2020. edn.. Edited by Schwabe U, Ludwig W-D. Berlin, Heidelberg: Springer Berlin Heidelberg; 2020: 3-41

[19] Bitter K, Schlender JF, Woltersdorf R. Arzneimittelinteraktionen im Alter. Internist (Berl) 2016; 57: 728-734

[20] Tiemann M, Mohokum M. Demografischer Wandel, Krankheitspanorama, Multimorbidität und Mortalität in Deutschland. In: Prävention und Gesundheitsförderung. edn. Edited by Tiemann M, Mohokum MBerlin, Heidelberg; Springer Berlin Heidelberg; 2020: 1-9

[21] Institut für Handelsforschung (IFH Köln): Apokix-Ergebnisse-November 2019 https://www.ifhkoeln.de/apokix-november-wie-digital-sinddeutsche-apotheken/ (abgerufen am 12.01.2021) 